\title{
Dapagliflozin Added to Glimepiride in Patients with Type 2 Diabetes Mellitus Sustains Glycemic Control and Weight Loss Over 48 Weeks: A Randomized, Double-Blind, Parallel-Group, Placebo-Controlled Trial
}

\author{
Krzysztof Strojek • Kun-Ho Yoon • Veronika Hruba · Jennifer Sugg • \\ Anna Maria Langkilde $\cdot$ Shamik Parikh \\ To view enhanced content go to www.diabetestherapy-open.com \\ Received: April 3, 2014 / Published online: June 12, 2014 \\ (C) The Author(s) 2014. This article is published with open access at Springerlink.com
}

\section{ABSTRACT}

Introduction: Maintenance of drug efficacy and safety over the long term is important to investigate for progressive conditions like type 2 diabetes mellitus (T2DM). This study aimed to evaluate whether efficacy of dapagliflozin added

Trial Registration: ClinicalTrials.gov \#NCT00680745.

Electronic supplementary material The online version of this article (doi:10.1007/s13300-014-0072-0) contains supplementary material, which is available to authorized users.

\section{K. Strojek $(\bowtie)$}

Department of Internal Diseases, Diabetology and Cardiometabolic Diseases, Silesian Centre for Heart Diseases, Silesian Medical University, Zabrze, Poland e-mail: KSTROJEK@sum.edu.pl

\section{K.-H. Yoon}

Department of Endocrinology and Metabolism, The Catholic University of Korea,

Seoul, Republic of Korea

V. Hruba

Clinical Development, AstraZeneca, Prague,

Czech Republic

J. Sugg $\cdot$ S. Parikh

Clinical Development, AstraZeneca, Wilmington, DE, USA

A. M. Langkilde

Clinical Development, AstraZeneca, Mölndal, Sweden to glimepiride observed at 24 weeks was maintained at 48 weeks, and to provide further safety and tolerability data in patients with T2DM.

Methods: This 24-week randomized, double-blind, parallel-group, placebo-controlled trial with a 24-week double-blind extension period enrolled adults whose T2DM was inadequately controlled [glycated hemoglobin $\left.\left(\mathrm{HbA}_{1 \mathrm{c}}\right) \quad 7.0-10.0 \%\right]$ on sulfonylurea monotherapy. Patients were randomized to placebo $(n=146)$ or dapagliflozin $2.5 \mathrm{mg}$ $(n=154), 5 \mathrm{mg}(n=145)$, or $10 \mathrm{mg}(n=151)$ per day added to open-label glimepiride $4 \mathrm{mg} /$ day.

Results: In total, 519 patients (87.1\%) completed the study. At 48 weeks, $\mathrm{HbA}_{1 \mathrm{c}}$ adjusted mean changes from baseline for the placebo versus dapagliflozin 2.5/5/10-mg groups were $-0.04 \%$ versus $-0.41 \%,-0.56 \%$ and $-0.73 \%$, respectively. There were no meaningful differences in $\mathrm{HbA}_{1 \mathrm{c}}$ changes from baseline from 24 to 48 weeks, indicating that glycemic efficacy was maintained. Improvements in fasting plasma glucose and post-challenge plasma glucose were also observed with dapagliflozin over 48 weeks. Dapagliflozin 2.5/5/10 mg produced sustained 
reductions in weight $(-1.36 /-1.54 /-2.41 \mathrm{~kg})$ versus placebo $(-0.77 \mathrm{~kg})$. Adjusted mean reductions from baseline in systolic blood pressure were also greater than placebo for all dapagliflozin doses. In the placebo versus dapagliflozin groups, serious adverse events were $8.9 \%$ versus $8.6-11.0 \%$, hypoglycemic events were $6.8 \%$ versus $9.7-11.3 \%$, and events suggestive of genital infection were $1.4 \%$ versus $5.2-8.6 \%$.

Conclusion: Dapagliflozin added to glimepiride improved glycemic control and body weight, with short-term findings maintained during the study's extension period. Therapy was generally well tolerated over 48 weeks; hypoglycemic events and events suggestive of genital infection were reported more often in patients receiving dapagliflozin.

Keywords: Dapagliflozin;

Glycemic control;

Glimepiride;

co-transporter 2 (SGLT2) inhibitor;

Sulfonylureas; Type 2 diabetes mellitus

\section{INTRODUCTION}

Sulfonylureas are commonly used in the medical management of type 2 diabetes mellitus (T2DM) after diet and exercise have failed; however, their long-term effectiveness is poor $[1,2]$. Loss of efficacy with glucose-lowering agents over time, particularly with sulfonylureas, is a consequence of progressive worsening of diabetes, characterized by loss of $\beta$-cell function [3]. Dapagliflozin, a competitive, reversible, and highly selective inhibitor of sodium-glucose co-transporter 2 (SGLT2), increases urinary glucose excretion and reduces hyperglycemia independently of insulin secretion or action [4-7]. Thus, its efficacy is expected to be complementary to that of agents with insulin-dependent mechanisms of action. We previously published results from the first 24 weeks of a double-blind study in patients with T2DM inadequately controlled on sulfonylurea monotherapy, which showed that dapagliflozin added to glimepiride significantly reduced glycated hemoglobin $\left(\mathrm{HbA}_{1 \mathrm{c}}\right)$, fasting plasma glucose (FPG), post-challenge plasma glucose, and weight compared with placebo added to glimepiride [8]. When considering newer, insulin-independent treatments such as the SGLT2 inhibitor, dapagliflozin, maintenance of efficacy achieved in the short-term over a longer period of time is important to establish, particularly for chronic, progressive conditions such as T2DM where some conventional, insulin-dependent therapies are associated with loss of efficacy over time $[2,9]$.

The objectives of the present analysis were to evaluate whether efficacy of dapagliflozin at 24 weeks was maintained after 48 weeks of treatment and to provide further safety and tolerability data with dapagliflozin.

\section{MATERIALS AND METHODS}

\section{Patients}

Patients aged $\geq 18$ years with inadequately controlled T2DM (defined as $\mathrm{HbA}_{1 \mathrm{c}} \geq 7.0 \%$ and $\leq 10.0 \%$ ), and who were receiving a stable dose of sulfonylurea monotherapy at a level of at least half the maximum recommended dose for at least 8 weeks prior to enrollment, were eligible for inclusion. In view of the observation that the glucose-lowering effects of sulfonylureas start to reach a plateau at half-maximal doses, and higher doses are not recommended because of excessive risk of 
hypoglycemia [2], the patients included in this study were highly likely to have been exposed to a maximally effective dose of glimepiride. In addition, patients had to have an FPG $\leq 15 \mathrm{mmol} / \mathrm{L}$ and fasting C-peptide $\geq 0.33 \mathrm{nmol} / \mathrm{L}$.

\section{Study Design}

This was a 48-week randomized, double-blind, parallel-group, placebo-controlled, phase III trial that included a 24-week double-blind, short-term treatment period with a 24 -week site- and subject-blinded, long-term extension period conducted from April 2008 to November 2009 at 84 sites in Europe and Asia. The study complied with the Declaration of Helsinki and the International Conference on Harmonisation/Good Clinical Practice Guidelines (July 1996). It was approved by institutional review boards and independent ethics committees for participating centers and is registered with ClinicalTrials.gov (NCT00680745). All participants provided informed consent. Full details of the study methods of the 24-week short-term, double-blind treatment period have been previously published [8]. A brief summary of study methods is as follows.

\section{Study Treatments and Interventions}

Eligible patients either continued with, or were switched to, open-label glimepiride $4 \mathrm{mg}$ /day during the 8-week lead-in period. During a 1-week qualification period, all inclusion/exclusion criteria were reviewed in those patients switched to glimepiride $4 \mathrm{mg} /$ day, as previously described [8]. Those patients still meeting the study eligibility criteria were randomized to receive double-blind dapagliflozin 2.5 , 5, or $10 \mathrm{mg}$, or placebo, taken orally once daily before the first meal of the day, while continuing open-label glimepiride $4 \mathrm{mg} /$ day. Open-label glimepiride could be downtitrated to $2 \mathrm{mg}$ or discontinued to mitigate recurrent hypoglycemic events at the discretion of the investigator. However, no uptitration was allowed.

Patients with inadequate glycemic control during the treatment period remained in the trial but received open-label rescue therapy with metformin or pioglitazone, or rosiglitazone (if pioglitazone was unavailable). The following were the progressively more stringent glycemic levels for defining inadequate glycemic control and eligibility for rescue therapy: a central laboratory FPG (confirmed on a second measurement within 3-5 days) $>15 \mathrm{mmol} / \mathrm{L}$ (weeks 4-8); an FPG $>13.2 \mathrm{mmol} / \mathrm{L}$ (weeks 8-12); or an FPG $>11.1 \mathrm{mmol} / \mathrm{L}$ (weeks 12-24). Patients with an $\mathrm{HbA}_{1 \mathrm{c}}$ level $>8.0 \%$ for a continuous 12-week period, despite maximum tolerated dose of rescue therapy, discontinued the trial. From 24 to 48 weeks, the criterion for rescue was a central laboratory $\mathrm{HbA}_{1 \mathrm{c}}$ value $\geq 8.0 \%$.

All patients received dietary and lifestyle counseling. In addition, patients with a body mass index of $\geq 27 \mathrm{~kg} / \mathrm{m}^{2}$ received advice concerning reducing caloric intake and exercise.

Details of the randomization schedule and methods for maintaining allocation concealment have been previously published [8].

\section{Outcome Measures}

The primary efficacy assessment was at 24 weeks. Specified exploratory efficacy assessments included: change in $\mathrm{HbA}_{1 \mathrm{c}}$ percentage from baseline to week 48; proportions of patients achieving a therapeutic glycemic response (defined as $\mathrm{HbA}_{1 \mathrm{c}}<7.0 \%$ and $\leq 6.5 \%$ at week 48 ); change in total body weight 
from baseline to week 48; change from baseline to week 48 in 2-hour post-challenge plasma glucose rise in response to an oral glucose tolerance test (OGTT); change in FPG from baseline to week 48; Diabetes Treatment Satisfaction Questionnaire (DTSQ) status at week 48; proportions of patients receiving rescue therapy for failing to reach prespecified glycemic targets or discontinuing for lack of efficacy; seated systolic and diastolic blood pressure (SBP and DBP, respectively); and lipid parameters. Details of OGTT administration have been published previously [8].

Safety and tolerability were evaluated by assessment of adverse events (AEs), laboratory values, electrocardiogram, pulse rate, blood pressure, hypoglycemic events, and calculated creatinine clearance as previously published [8]. A prespecified list of Medical Dictionary for Regulatory Activities (MedDRA) version 13.0 preferred terms identified signs, symptoms, and other reports suggestive of urinary tract infection (UTI) and genital infection in the database. This list included terms for nonspecific signs and symptoms suggestive of genital infection (e.g., genital pruritus, vulvovaginal pruritus) as well as terms for clinical infection (e.g., vaginal infection), but did not include terms for sexually transmitted diseases. Patients reported these events both spontaneously and in response to proactive questioning by the investigators during study visits.

\section{Statistical Analysis}

The continuous efficacy analyses over 48 weeks were based on longitudinal repeated measures analyses using "direct likelihood" without imputation of missing data. The SAS procedure PROC MIXED (SAS Institute Inc, Cary, NC, USA) was used, and model terms included the fixed categorical effects of treatment, week and treatment-by-week interaction, and the continuous fixed covariates of baseline measurement and baseline measurement-by-week interaction. No statistical hypothesis testing was performed for the combined short-term plus extension periods from baseline to week 48, but point estimates and $95 \%$ confidence intervals (CIs) were provided. Categorical efficacy variables were analyzed using logistic regression methodology from Zhang et al. [10] with adjustment for baseline value. Patients with missing data were included in these analyses and considered non-responders. The Kaplan-Meier method was used to analyze time to onset of rescue therapy for failing to achieve prespecified glycemic targets or patient discontinuation due to poor glycemic control. Efficacy analyses exclude data after rescue, unless otherwise stated. Safety analyses include data after rescue.

Definitions of the full analysis set and safety analysis set and sample size calculations were as previously published [8]. Safety data were summarized with the safety analysis set using descriptive statistics.

\section{RESULTS}

\section{Patients}

Detailed demographic and clinical characteristics at baseline were previously reported and balanced across the treatment groups [8]. Key baseline characteristics are summarized in Table 1 . The majority of randomized patients completed the short-term and the extension periods $[519$ patients $(87.1 \%)]$, with the most common reasons for discontinuation being withdrawal of consent 
Table 1 Demographic and baseline characteristics of the full analysis set

\begin{tabular}{|c|c|c|c|c|}
\hline Characteristic & $\begin{array}{l}\text { Placebo + } \\
\text { glimepiride } \\
(N=145)\end{array}$ & $\begin{array}{l}\text { Dapagliflozin } 2.5 \mathrm{mg}+ \\
\text { glimepiride }(N=154)\end{array}$ & $\begin{array}{l}\text { Dapagliflozin } 5 \mathrm{mg}+ \\
\text { glimepiride }(N=142)\end{array}$ & $\begin{array}{l}\text { Dapagliflozin } 10 \mathrm{mg}+ \\
\text { glimepiride }(N=151)\end{array}$ \\
\hline Age (years) & $60.3 \pm 10.2$ & $59.9 \pm 10.1$ & $60.2 \pm 9.7$ & $58.9 \pm 8.3$ \\
\hline $\begin{array}{l}\text { Type } 2 \text { diabetes } \\
\text { duration (years) }\end{array}$ & $7.4 \pm 5.7$ & $7.7 \pm 6.0$ & $7.4 \pm 5.7$ & $7.2 \pm 5.5$ \\
\hline Male (\%) & 49.0 & 50.0 & 50.0 & 43.7 \\
\hline $\operatorname{BMI}\left(\mathrm{kg} / \mathrm{m}^{2}\right)$ & $29.7 \pm 4.6$ & $30.0 \pm 5.1$ & $29.8 \pm 5.2$ & $29.8 \pm 5.6$ \\
\hline $\mathrm{HbA}_{1 \mathrm{c}}(\%)$ & $8.15 \pm 0.74$ & $8.11 \pm 0.75$ & $8.12 \pm 0.78$ & $8.07 \pm 0.79$ \\
\hline FPG $(\mathrm{mmol} / \mathrm{L})$ & $9.58 \pm 2.07$ & $9.56 \pm 2.13$ & $9.68 \pm 2.12$ & $9.55 \pm 2.04$ \\
\hline $\begin{array}{l}\text { Prior history of } \\
\operatorname{CVD}^{\mathrm{a}}(\%)\end{array}$ & 37.9 & 36.4 & 38.7 & 30.5 \\
\hline Hypertension (\%) & 80.0 & 70.1 & 70.4 & 74.8 \\
\hline $\begin{array}{l}\text { Overall DTSQ } \\
\text { score } \\
\text { (maximum 36) }\end{array}$ & $28.4 \pm 7.1$ & $27.8 \pm 6.0$ & $27.4 \pm 6.0$ & $27.6 \pm 6.5$ \\
\hline
\end{tabular}

All data are mean \pm standard deviation, unless otherwise specified

$B M I$ body mass index, CVD cardiovascular disease, DTSQ Diabetes Treatment Satisfaction Questionnaire, FPG fasting plasma glucose, $H b A_{1 c}$ glycated hemoglobin

${ }^{a}$ At least one event (excluding patients with a history of hypertension only)

and AEs [Supplementary Figure S1 in the electronic supplementary material (ESM)].

\section{Efficacy Variables}

Adjusted mean changes in $\mathrm{HbA}_{1 \mathrm{c}}$ from baseline at 48 weeks after dapagliflozin $2.5,5$, and $10 \mathrm{mg}$ were added to glimepiride were -0.41 (95\% CI $-0.56,-0.26),-0.56$ (95\% CI $-0.71,-0.41)$, and -0.73 percentage points $(95 \%$ CI -0.87 , $-0.59)$, respectively, versus -0.04 percentage points $(95 \% \mathrm{CI}-0.21,0.14)$ in the placebo plus glimepiride group (excluding data after rescue therapy; Fig. 1a). Differences between the dapagliflozin and placebo groups were statistically significant at 24 weeks [8] and were maintained at 48 weeks with placebocorrected reductions of -0.37 percentage points $(95 \%$ CI $-0.60,-0.14)$ in the dapagliflozin 2.5-mg group, -0.53 percentage points $(95 \% \mathrm{CI}-0.75,-0.30)$ in the $5-\mathrm{mg}$ group, and -0.70 percentage points $(95 \% \mathrm{CI}$ $-0.92,-0.47)$ in the 10-mg group. In analyses that included data after rescue therapy, $\mathrm{HbA}_{1 \mathrm{c}}$ changes with dapagliflozin added to glimepiride were similar (Supplementary Figure S2 in the ESM). Comparisons of 24-week versus 48 -week $\mathrm{HbA}_{1 \mathrm{c}}$ change from baseline values (excluding data after rescue therapy) revealed no substantive differences in placebo-corrected reductions between these time points (Supplementary Table S1 in the ESM).

The proportion of patients achieving an $\mathrm{HbA}_{1 \mathrm{c}}<7.0 \%$ or $<6.5 \%$ was significantly increased with dapagliflozin 5 and $10 \mathrm{mg}$ versus placebo at 24 weeks [8]. This was maintained at week 48 , with the proportions of patients achieving an $\mathrm{HbA}_{1 \mathrm{c}}<7.0 \%$ higher in 

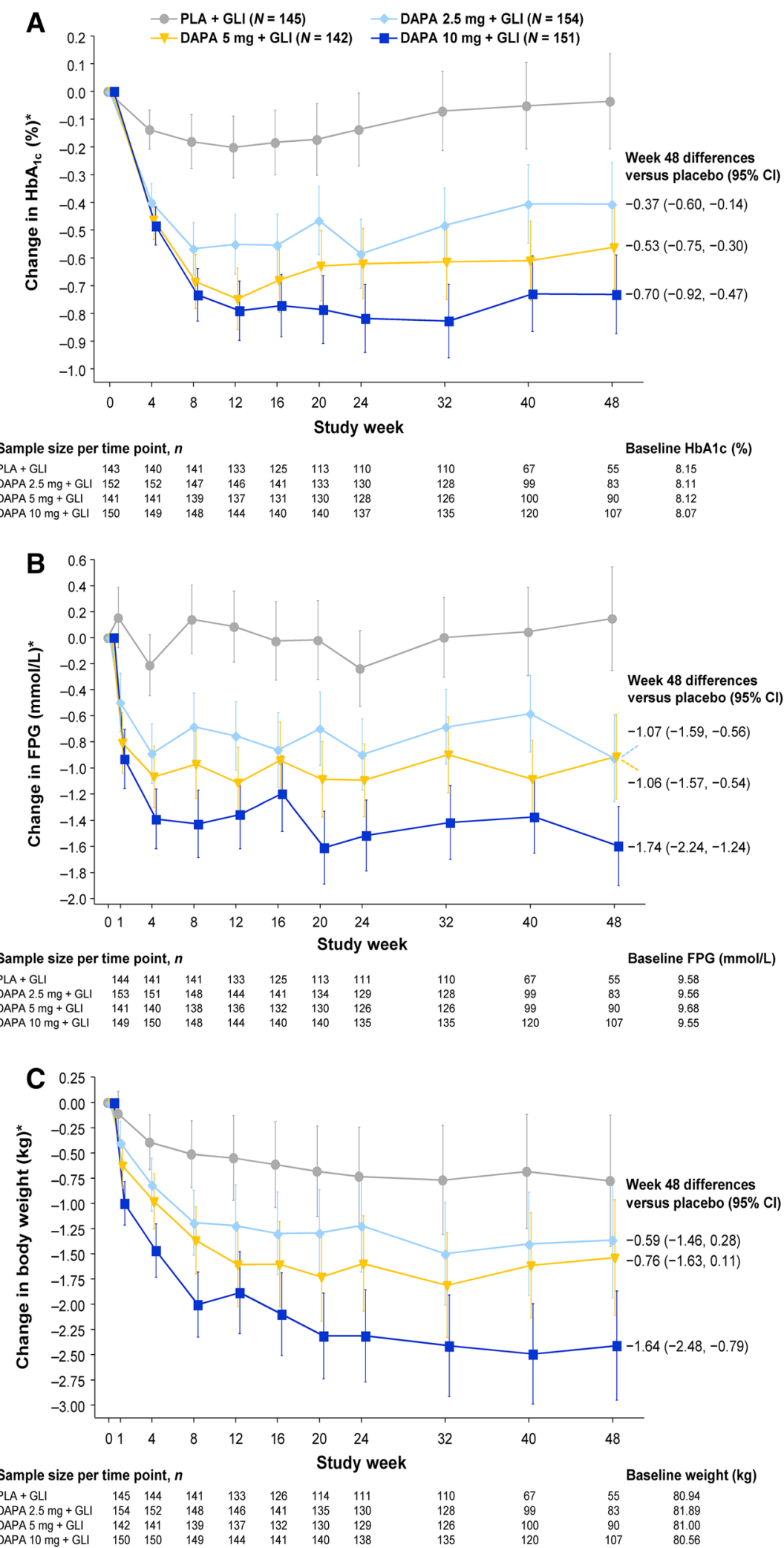
4Fig. 1 Change in a $\mathrm{HbA}_{1 \mathrm{c}}$ (\%), b FPG, and $\mathbf{c}$ body weight with treatment over 48 weeks (full analysis set) excluding data after rescue therapy. ${ }^{*}$ Data are adjusted mean changes from baseline $\pm 95 \%$ confidence interval derived from a repeated-measures mixed model. $N$ is the number of patients in the full analysis set, and $n$ is the number of patients in the full analysis set with non-missing baseline and week $(t)$ values. Treatment group symbols are shifted horizontally to prevent error bar overlapping. DAPA dapagliflozin, $C I$ Confidence interval, GLI glimepiride, $H b A_{1 c}$ glycated hemoglobin, PLA placebo

the dapagliflozin 2.5-, 5-, and 10-mg groups (15.6\%, 28.2\%, and 30.5\%, respectively) than in the placebo group (10.3\%). Corresponding values for proportions of patients achieving an $\mathrm{HbA}_{1 \mathrm{c}}<6.5 \%$ at week 48 were 9.7\%, 14.8\%, $16.6 \%$, and $2.8 \%$, respectively.

Dapagliflozin also produced sustained reductions in FPG of up to $1.60 \mathrm{mmol} / \mathrm{L}$ at 48 weeks in the 10-mg group (Fig. 1b). Adjusted mean changes in FPG from baseline at 48 weeks after dapagliflozin $2.5,5$, and $10 \mathrm{mg}$ were added to glimepiride were -0.93 (95\% CI -1.26 , $-0.59),-0.92(95 \%$ CI $-1.24,-0.59)$, and $-1.60 \mathrm{mmol} / \mathrm{L} \quad(95 \% \quad \mathrm{CI} \quad-1.90,-1.29)$, respectively, versus $0.14 \mathrm{mmol} / \mathrm{L} \quad(95 \% \quad \mathrm{CI}$ $-0.25,0.54)$ in the placebo plus glimepiride group (excluding data after rescue therapy). Differences between the dapagliflozin 5- and 10-mg groups and the placebo group were statistically significant at 24 weeks [8], and these differences were maintained at 48 weeks with placebo-corrected reductions of $-1.06 \mathrm{mmol} / \mathrm{L} \quad(95 \% \mathrm{CI}-1.57,-0.54)$ and $-1.74 \mathrm{mmol} / \mathrm{L} \quad(95 \% \quad \mathrm{CI} \quad-2.24,-1.24)$, respectively.

The 2-h post-challenge plasma glucose rise in response to an OGTT was significantly reduced from baseline at 24 weeks in the dapagliflozin 5- and 10-mg groups [8], and these differences were maintained at 48 weeks with placebo-corrected reductions in adjusted mean change from baseline of $-1.38 \mathrm{mmol} / \mathrm{L}$ (95\% CI
$-2.49,-0.27)$ and $-1.20 \mathrm{mmol} / \mathrm{L}(95 \%$ CI $-2.26,-0.14)$, respectively.

Dapagliflozin produced sustained reductions in body weight of up to $2.41 \mathrm{~kg}$ at 48 weeks in the 10-mg group (Fig. 1c). Adjusted mean changes in body weight from baseline at 48 weeks after dapagliflozin $2.5,5$, and $10 \mathrm{mg}$ were added to glimepiride were -1.36 (95\% CI $-1.94,-0.79),-1.54$ (95\% CI $-2.11,-0.96)$, and $\quad-2.41 \mathrm{~kg} \quad(95 \% \quad \mathrm{CI} \quad-2.95,-1.87)$, respectively, versus $-0.77 \mathrm{~kg}(95 \% \mathrm{CI}-1.43$, -0.12 ) in the placebo plus glimepiride group (excluding data after rescue therapy). The differences between the dapagliflozin 5- and $10-\mathrm{mg}$ groups and the placebo group were statistically significant at 24 weeks [8]; and the difference in the dapagliflozin 10-mg group was maintained at 48 weeks with a placebo-corrected reduction of $-1.64 \mathrm{~kg}$ (95\% CI -2.48, -0.79).

Overall, fewer patients in the dapagliflozin 2.5-, 5-, and 10-mg groups discontinued or received rescue medication due to poor glycemic control over 48 weeks $(32.5 \%, 29.6 \%$, and $17.9 \%$, respectively) compared with the placebo group (53.1\%). The probability of discontinuation or rescue was lower in the dapagliflozin groups compared with the placebo group over the entire study period (Fig. 2). Only 7 patients were discontinued due to lack of efficacy: 3 in the placebo group $(2.1 \%), 2$ in the dapagliflozin 5-mg group (1.4\%), and 1 each in the dapagliflozin 2.5and $10-\mathrm{mg}$ groups $(0.6 \%$ and $0.7 \%$, respectively).

For seated SBP, adjusted mean reductions from baseline at week 48 were greater than placebo for all dapagliflozin doses $(-4.33$ to $-6.02 \mathrm{mmHg}$ ). For seated DBP, only the dapagliflozin 10-mg dose showed a meaningful reduction $(-2.94 \mathrm{mmHg})$ compared with placebo at week 48 (Supplementary Figure S3 


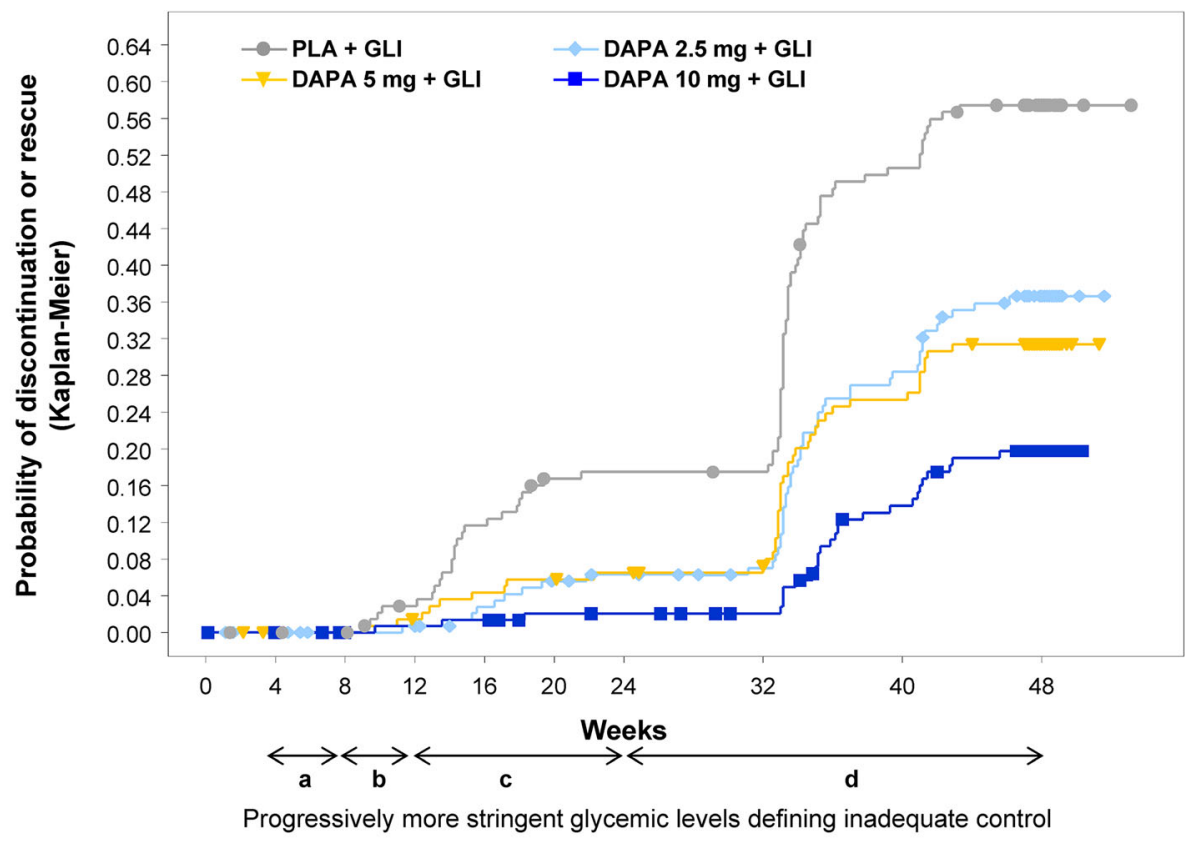

Number of patients at risk

$\begin{array}{llllllll}\text { PLA + GLI } & 145 & 144 & 142 & 133 & 121 & 112 & 111 \\ \text { DAPA 2.5 mg + GLI } & 154 & 151 & 147 & 145 & 139 & 134 & 131 \\ \text { DAPA 5 mg + GLI } & 142 & 140 & 139 & 136 & 132 & 130 & 128 \\ \text { DAPA 10 mg + GLI } & 151 & 150 & 147 & 145 & 144 & 140 & 139\end{array}$

Fig. 2 Probability of rescue therapy for failing to achieve prespecified glycemic targets or of discontinuation because of poor glycemic control over time. Symbols represent censored observations. Progressively more stringent glycemic levels for defining inadequate glycemic control and hence eligibility for rescue therapy were $a$ Central

in the ESM). No meaningful changes in heart rate were observed in any treatment group (mean change from baseline in seated heart rate was $0.6,-0.3,0.2$, and 1.6 beats per minute in the dapagliflozin 2.5, 5, 10-mg groups and placebo group, respectively).

At week 48, small percentage increases in high-density lipoprotein cholesterol and free fatty acids from baseline were seen in the dapagliflozin treatment groups compared with placebo, which were slightly more pronounced at week 48 than at week 24. For low-density lipoprotein cholesterol, there were no relevant changes from baseline to week 48; mean percent changes were $-0.6 \%(95 \%$ CI -6.7 , laboratory FPG (confirmed on a second measurement within 3-5 days) $>15 \mathrm{mmol} / \mathrm{L}$ during weeks $4-8 ; b$ an FPG $>13.2 \mathrm{mmol} / \mathrm{L}$ during weeks $8-12 ; c$ an $\mathrm{FPG}>11.1$ $\mathrm{mmol} / \mathrm{L}$ during weeks $12-24$; and $d$ central laboratory $\mathrm{HbA}_{1 \mathrm{c}} \geq 8.0 \%$ during weeks 24-48. DAPA dapagliflozin, FPG fasting plasma glucose, GLI glimepiride, PLA placebo

5.6) for placebo and 7.8\% (95\% CI 1.0, 14.6), $5.7 \%$ (95\% CI $-1.8,13.3)$, and $3.9 \%$ (95\% CI $-1.1,8.9$ ) for dapagliflozin 2.5-, 5-, and 10-mg groups, respectively. Similarly, for total cholesterol and triglycerides, no relevant changes from baseline to week 48 or from week 24 to week 48 were noted in any treatment group (Supplementary Table S2 in the ESM). Fasting C-peptide at baseline was above the normal reference range (0.17-0.83 $\mathrm{nmol} / \mathrm{L}$ ) across all groups (Supplementary Table S2); levels were reduced with dapagliflozin at week 24 and to a greater extent at week 48 . Fasting C-peptide at week 48 was reduced by -0.09 (95\% CI $-0.15,-0.03),-0.10$ (95\% CI 
$-0.20,0.01)$, and -0.07 (95\% CI $-0.14,-0.01)$ $\mathrm{nmol} / \mathrm{L}$ in the dapagliflozin 2.5-, 5-, and 10-mg groups, respectively, but to a lesser extent in the placebo group $[-0.01(95 \%$ CI $-0.14,0.12)$ $\mathrm{nmol} / \mathrm{L}]$.

Patients' overall mean DTSQ scores improved slightly from baseline at week 48, although values were similar in the dapagliflozin 2.5-, 5-, and 10-mg groups versus placebo; 3.5 (95\% CI 2.3, 4.6), 4.6 (95\% CI 3.2, $5.9)$, and 4.4 (95\% CI 3.1, 5.8), respectively, versus $2.2(95 \% \mathrm{CI} 0.5,3.9)$.

\section{Safety and Tolerability}

AEs and AEs leading to study discontinuation were balanced across the treatment groups (Table 2). The frequencies of serious AEs and AEs reported as drug-related were higher in the dapagliflozin groups compared with the placebo group (Table 2).

Serious AEs led to discontinuation of study medication in eight patients $[<2.2 \%$ of patients in each treatment group (Table 2)]: three in the placebo group (syncope, pulmonary edema, and multiple stenosis of the coronary arteries), three in the dapagliflozin 2.5-mg group (neutropenic fever, chronic lymphatic leukemia, and gastroduodenitis), one in the dapagliflozin 5-mg group (decompensation of diabetes mellitus), and one in the dapagliflozin $10-\mathrm{mg}$ group (stroke). Three deaths were reported, which were assessed as not related to study treatment. These included two deaths [ 1 in the dapagliflozin 2.5-mg group (cardiopulmonary arrest) and 1 in the 10-mg group (pulmonary embolism after ischemic stroke in a patient with a history of aortic valve replacement)] that occurred during the short-term period and have been previously described [8]. During the extension period, a 66-year-old male patient in the dapagliflozin 2.5-mg group died of an acute myocardial infarction.

Hypoglycemic events were reported more frequently in the dapagliflozin groups $(9.7-11.3 \%)$ than in the placebo group $(6.8 \%)$. However, no patients were discontinued due to hypoglycemia (Table 2), and most patients experienced their first event during the first 24 weeks of treatment (Supplementary Figure S4 in the ESM; excluding data after rescue, but potential downtitration/discontinuation of glimepiride should be taken into account when interpreting these results). There was only one major episode of hypoglycemia reported (dapagliflozin 2.5-mg group).

Events suggestive of vulvovaginitis, balanitis, and related genital infection were more frequently reported in all dapagliflozin groups compared with placebo, and their frequency increased with increasing dapagliflozin dose (Table 2). The majority of these patients were females, with most reporting the first event during the short-term period (Fig. 3a). Events suggestive of UTI were reported at similar rates across all treatment groups (Table 2), and most patients reported the first event during the short-term period (Fig. 3b). No kidney infections were reported. All of the events suggestive of genital infections or UTIs were of mild or moderate intensity, and only 1 patient receiving dapagliflozin $10 \mathrm{mg}$ discontinued the study due to an AE suggestive of UTI. Cultures were obtained only in a proportion of cases; thus, not all reports represented confirmed infections. Nevertheless, the majority of these events were treated with antimicrobial agents. For events suggestive of genital infection, 0 out of a total of 2 events in the placebo group and 29 out of a total of 40 events across the dapagliflozin groups were treated; repeated treatment because of inadequate response was 
Table 2 Overall summary of patients with an AE over 48 weeks (safety analysis set)

\begin{tabular}{|c|c|c|c|c|}
\hline Preferred term & $\begin{array}{l}\text { Placebo }+ \\
\text { glimepiride } \\
(N=146)\end{array}$ & $\begin{array}{l}\text { Dapagliflozin } 2.5 \mathrm{mg}+ \\
\text { glimepiride }(N=154)\end{array}$ & $\begin{array}{l}\text { Dapagliflozin } 5 \mathrm{mg}+ \\
\text { glimepiride }(N=145)\end{array}$ & $\begin{array}{l}\text { Dapagliflozin } 10 \mathrm{mg}+ \\
\text { glimepiride }(N=151)\end{array}$ \\
\hline \multicolumn{5}{|c|}{ Overall summary of patients with an AE, $n(\%)$} \\
\hline One or more $\mathrm{AE}$ & $81(55.5)$ & $90(58.4)$ & $88(60.7)$ & $89(58.9)$ \\
\hline $\begin{array}{l}\text { One or more drug- } \\
\text { related } \mathrm{AE}^{\mathrm{a}}\end{array}$ & $8(5.5)$ & $12(7.8)$ & $12(8.3)$ & $16(10.6)$ \\
\hline $\begin{array}{l}\text { AE leading to } \\
\text { discontinuation }\end{array}$ & $5(3.4)$ & $5(3.2)$ & $5(3.4)$ & $4(2.6)$ \\
\hline One or more $\mathrm{SAE}^{\mathrm{b}}$ & $13(8.9)$ & $16(10.4)$ & $16(11.0)$ & $13(8.6)$ \\
\hline $\begin{array}{l}\text { SAE leading to } \\
\text { discontinuation }\end{array}$ & $3(2.1)$ & $3(1.9)$ & $1(0.7)$ & $1(0.7)$ \\
\hline Deaths & $0(0.0)$ & $2(1.3)$ & $0(0.0)$ & $1(0.7)$ \\
\hline \multicolumn{5}{|c|}{ Patients with AEs of $\geq 3 \%$ in any group (by MedDRA preferred term), $n(\%)$} \\
\hline Nasopharyngitis & $10(6.8)$ & $6(3.9)$ & $11(7.6)$ & $10(6.6)$ \\
\hline $\begin{array}{l}\text { Upper respiratory } \\
\text { tract infection }\end{array}$ & $4(2.7)$ & $5(3.2)$ & $7(4.8)$ & $7(4.6)$ \\
\hline $\mathrm{UTI}^{\mathrm{c}}$ & $7(4.8)$ & $5(3.2)$ & $4(2.8)$ & $6(4.0)$ \\
\hline Bronchitis & $1(0.7)$ & $2(1.3)$ & $4(2.8)$ & $5(3.3)$ \\
\hline Arthralgia & $5(3.4)$ & $8(5.2)$ & $0(0.0)$ & $3(2.0)$ \\
\hline Diarrhea & $7(4.8)$ & $4(2.6)$ & $4(2.8)$ & $1(0.7)$ \\
\hline Dyspepsia & $5(3.4)$ & $2(1.3)$ & $4(2.8)$ & $1(0.7)$ \\
\hline Cough & $2(1.4)$ & $1(0.6)$ & $2(1.4)$ & $5(3.3)$ \\
\hline Dyslipidemia & $0(0.0)$ & $5(3.2)$ & $2(1.4)$ & $4(2.6)$ \\
\hline Hypertension & $10(6.8)$ & $9(5.8)$ & $5(3.4)$ & $3(2.0)$ \\
\hline \multicolumn{5}{|c|}{ Patients with a special interest AE, $n$ (\%) } \\
\hline $\begin{array}{l}\text { One or more } \\
\text { hypoglycemic } \\
\text { events }{ }^{\mathrm{d}, \mathrm{e}}\end{array}$ & $10(6.8)$ & $15(9.7)$ & $15(10.3)$ & $17(11.3)$ \\
\hline Major episode & $0(0.0)$ & $1(0.6)$ & $0(0.0)$ & $0(0.0)$ \\
\hline Minor episode & $4(2.7)$ & $5(3.2)$ & $12(8.3)$ & $13(8.6)$ \\
\hline Other episode & $7(4.8)$ & $11(7.1)$ & $5(3.4)$ & $5(3.3)$ \\
\hline
\end{tabular}


Table 2 continued

\begin{tabular}{|c|c|c|c|c|}
\hline Preferred term & $\begin{array}{l}\text { Placebo }+ \\
\text { glimepiride } \\
(N=146)\end{array}$ & $\begin{array}{l}\text { Dapagliflozin } 2.5 \mathrm{mg}+ \\
\text { glimepiride }(N=154)\end{array}$ & $\begin{array}{l}\text { Dapagliflozin } 5 \mathrm{mg}+ \\
\text { glimepiride }(N=145)\end{array}$ & $\begin{array}{l}\text { Dapagliflozin } 10 \mathrm{mg}+ \\
\text { glimepiride }(N=151)\end{array}$ \\
\hline \multicolumn{5}{|c|}{$\begin{array}{l}\text { Events suggestive of } \\
\text { genital infection }\end{array}$} \\
\hline Total & $2(1.4)$ & $8(5.2)$ & $9(6.2)$ & $13(8.6)$ \\
\hline Males & $0 / 71(0.0)$ & $1 / 77(1.3)$ & $2 / 72(2.8)$ & $5 / 66(7.6)$ \\
\hline Females & $2 / 75(2.7)$ & 7/77 (9.1) & $7 / 73(9.6)$ & $8 / 85(9.4)$ \\
\hline \multicolumn{5}{|c|}{$\begin{array}{l}\text { Events suggestive of } \\
\text { UTI }^{f}\end{array}$} \\
\hline Total & $11(7.5)$ & $7(4.5)$ & $11(7.6)$ & $12(7.9)$ \\
\hline Males & $1 / 71(1.4)$ & $1 / 77(1.3)$ & $4 / 72(5.6)$ & $4 / 66(6.1)$ \\
\hline Females & $10 / 75(13.3)$ & 6/77 (7.8) & $7 / 73(9.6)$ & $8 / 85(9.4)^{g}$ \\
\hline $\begin{array}{l}\text { Renal impairment/ } \\
\text { failure }^{\mathrm{h}}\end{array}$ & $3(2.1)^{\mathrm{i}}$ & $1(0.6)^{\mathrm{g}, \mathrm{j}}$ & $1(0.7)^{\mathrm{k}}$ & $0(0.0)$ \\
\hline $\begin{array}{l}\text { Hypotension/ } \\
\text { dehydration/ } \\
\text { hypovolemia } \mathrm{d}, \mathrm{h}\end{array}$ & $0(0.0)$ & $1(0.6)$ & $0(0.0)$ & $1(0.7)$ \\
\hline
\end{tabular}

$N$ is the number of patients in the safety analysis set and includes data after rescue therapy

$A E$ adverse event, MedDRA Medical Dictionary for Regulatory Activities, SAE serious adverse event, UTI Urinary tract infection

a Events with certain, probable, possible, or unknown relation to study drug were deemed to be drug-related AEs

b No SAE was assessed as drug-related

c Based on definitive Medical Dictionary for Regulatory Activities preferred terms

d None led to study discontinuation

e Includes hypoglycemic events with onset on or after the first date of double-blind treatment, and on or prior to the last day of short-term or long-term double-blind treatment, plus 4 days or up to end of treatment visit if earlier. Major episode defined as a symptomatic episode requiring external (third party) assistance due to severe impairment in consciousness or behavior with a capillary or plasma glucose value $<3 \mathrm{mmol} / \mathrm{L}$ and prompt recovery after glucose or glucagon administration. Minor episode defined as either a symptomatic episode with a capillary or plasma glucose measurement $<3.5 \mathrm{mmol} / \mathrm{L}$, regardless of need for external assistance, or an asymptomatic capillary or plasma glucose measurement $<3.5 \mathrm{mmol} / \mathrm{L}$ that did not qualify as a major episode. Other episodes of hypoglycemia defined as suggestive episode reported but not meeting the criteria for major or minor episodes

$\mathrm{f}$ Events suggestive of genital infection or UTI were identified in the database using the prespecified lists of preferred terms. These events included signs, symptoms, and other reports suggestive of genital infection or UTI, as well as definitive terms for genital infection or UTI

g One patient discontinued the study

$\mathrm{h}$ These events were also identified in the database using prespecified lists of preferred terms, which also included, for example, laboratory values such as serum creatinine

i Two renal impairment and one renal failure

j Renal impairment

k Obstructive uropathy 
rarely required across the dapagliflozin groups (4/40). For events suggestive of UTIs, 18 out of a total of 19 events in the placebo group and 26 out of a total of 32 events across the dapagliflozin groups were treated; repeated treatment because of inadequate response was rarely required $(0 / 19$ in the placebo group and 2/32 across the dapagliflozin groups).

AE reports of neoplasms (benign, malignant, and unspecified) occurred in $1.3-3.9 \%$ of patients in the dapagliflozin groups compared with $1.4 \%$ of patients in the placebo group (Supplementary Table S3 in the ESM).

Overall, changes in clinical laboratory parameters from baseline to week 48 were small in magnitude. There were small mean increases in hematocrit $(2.26-2.53 \%)$ in the dapagliflozin groups that were not associated with thromboembolic AEs. A small increase in blood urea nitrogen $(0.39-0.68 \mathrm{mmol} / \mathrm{L})$ in the dapagliflozin groups was not associated with any worsening of renal function as evidenced
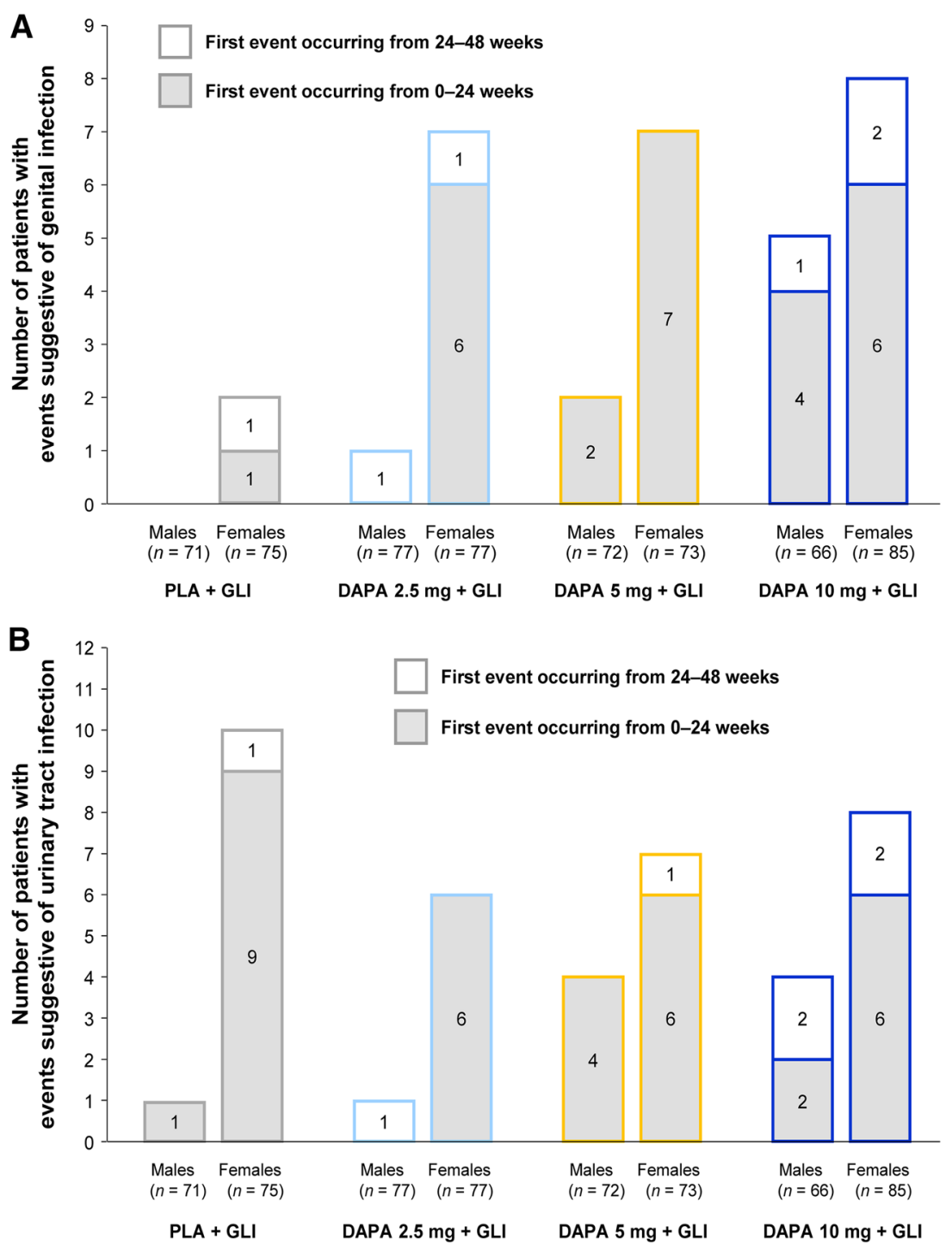

Fig. 3 Patients with events suggestive of a genital infections and $\mathbf{b}$ urinary tract infections (safety analysis set and include data after rescue therapy). DAPA dapagliflozin, GLI glimepiride, PLA placebo 
by the lack of any meaningful changes in serum creatinine, calculated creatinine clearance, estimated glomerular filtration rate, and serum cystatin-C (Supplementary Table S4 in the ESM).

\section{DISCUSSION}

The present study showed that the glycemic efficacy of dapagliflozin appeared to be dose dependent. The glycemic effect of dapagliflozin versus placebo was evident at the first study visit; a sustained, clinically meaningful effect was demonstrated from 12 to 48 weeks in the dapagliflozin 5-mg group and from 8 to 48 weeks in the 10-mg group. Moreover, the placebo-corrected reductions in $\mathrm{HbA}_{1 \mathrm{c}}$ demonstrated with all dapagliflozin doses at 24 weeks [8] were maintained over 48 weeks. In addition to these changes in $\mathrm{HbA}_{1 \mathrm{c}}$, dapagliflozin treatment was associated with sustained improvements in FPG over 48 weeks and reductions in postprandial plasma glucose rise in response to an OGTT at 24 weeks [8] and 48 weeks.

The proportion of patients requiring rescue medication or discontinuing due to lack of glycemic control was lower in the dapagliflozin groups compared with placebo, with markedly greater differences seen between the dapagliflozin and placebo groups at the end of the extension period than were noted at the end of the short-term period [8]. A sharp increase in the probability of rescue or discontinuation due to lack of glycemic control was evident after week 32 in all treatment groups (Fig. 2), which can be explained by the change in criteria for rescue therapy from an FPG $>11.0 \mathrm{mmol} / \mathrm{L}$ during the short-term treatment period to an $\mathrm{HbA}_{1 \mathrm{c}} \geq 8.0 \%$ during the extension period.

Regarding additional clinical benefits with dapagliflozin treatment, sustained reductions in body weight and SBP were noted over 48 weeks, consistent with findings observed in other clinical studies of dapagliflozin [11-13]. Owing to the insulin-dependent mechanism of action of sulfonylureas, these agents are often associated with weight gain [14]. The addition of dapagliflozin to sulfonylurea therapy has the potential, therefore, to not only improve glycemic efficacy, but also to mitigate sulfonylurea-associated weight gain. Reductions in SBP have been shown to reduce the incidence of cardiovascular events in patients with hypertension $[15,16]$ and in patients with T2DM $[17,18]$, the majority of whom also have hypertension $[19,20]$. Whether the modest reductions in SBP observed with dapagliflozin therapy translate into longer-term reductions in cardiovascular risk await results from the prospective DECLARE study (Clinicaltrials.gov \#NCT01730534) [21].

Overall, dapagliflozin was well tolerated. Although hypoglycemic events were more frequently reported in patients treated with dapagliflozin than with placebo, most first events occurred within the first 24 weeks of treatment. An increase in such events is commonly observed when glucose-lowering therapies with a low intrinsic potential to cause hypoglycemia are added to sulfonylurea therapy [22].

A higher proportion of patients treated with dapagliflozin (mostly female) reported events suggestive of vulvovaginitis, balanitis, and related genital infection compared with placebo. Although patients with diabetes are at increased risk of common infections, including genital infections [23-27], an increase in rates of events suggestive of genital infection has been consistently reported in clinical studies of dapagliflozin and canagliflozin [28-31]. Most of these events occurred during the first 24 weeks, were of mild or moderate intensity, responded to routine management, and rarely 
led to study discontinuation. Events suggestive of UTI occurred in similar proportions of patients in the dapagliflozin and placebo groups, in line with similar findings of other SGLT2 inhibitor therapies in patients with T2DM [32]. Findings reported in the present study were also similar to pooled analyses of 12 randomized, placebo-controlled trials of dapagliflozin as monotherapy or add-on therapy, which assessed the occurrence of genital infections and UTIs over 24 weeks [33, 34]. These pooled analyses also demonstrated that when actual clinical diagnoses of genital infections and UTIs (as opposed to events suggestive of) were considered, the proportions of patients with an event decreased across groups.

No deterioration of renal function was noted in the dapagliflozin groups. Although dapagliflozin was associated with modest rises in hematocrit and blood urea nitrogen, there were no changes in serum creatinine, creatinine clearance, estimated glomerular filtration rate, or serum cystatin-C (Supplementary Table S3). Furthermore, there was no increase in the proportions of patients experiencing AEs of renal impairment/failure, or AEs of hypotension/dehydration/hypovolemia (Table 2).

The decrease from baseline in fasting C-peptide to near normal levels observed with dapagliflozin in this study is interesting, given suggestions that high levels may be a marker for hyperinsulinemia and insulin resistance [32]. Theoretically, as the mechanism of action of dapagliflozin is independent of insulin secretion, the reduction in fasting C-peptide observed with treatment may be a result of reduced stress on $\beta$-cells, which may ultimately improve $\beta$-cell function over the long term. Indeed, a recent, small-scale study of 11 patients with T2DM demonstrated that lowering plasma glucose concentration with dapagliflozin improved insulin resistance and $\beta$-cell function [35]. Further in-depth investigation, outside the scope of the present study, is required to comprehensively examine SGLT2-facilitated relief of glucotoxicity in a clinical setting.

In our study, the proportion of patients with neoplasms (benign, malignant, and unspecified) was fractionally higher in the dapagliflozin groups compared with placebo, but no apparent anatomical pattern was noted. An analysis of $>4,500$ patients included in the dapagliflozin phase IIb/III database has indicated that overall incidence rates for malignant and unspecified tumors (per 100 patient-years) were similar for dapagliflozin (1.34) versus control treatments (1.39) [36]. However, trends in specific malignancies will continue to be evaluated in further observational and randomized clinical studies.

Limitations of the study design have been published previously [8]. Although excluding data after rescue therapy allows changes attributable to the investigational treatment to be evaluated (Fig. 1a), the progressive loss of patient numbers from the analysis limits the confidence of these findings. Display of all patient data, including after rescue therapy (Supplementary Figure S2), shows that a high number of patients completed the study. Although including all treated patients comes closer to actual clinical practice, interpretation of the results is confounded by changes in $\mathrm{HbA}_{1 \mathrm{c}}$ and other variables consequent to the addition of rescue medication. We have reported both analyses to evaluate the robustness of dapagliflozin treatment effects and found that the adjusted mean changes from baseline in $\mathrm{HbA}_{1 \mathrm{c}}$ were similar between the two analytic strategies. 


\section{CONCLUSION}

Loss of efficacy over time is observed with some treatments for T2DM $[2,9]$. To our knowledge, this is the longest study to date of an SGLT2 inhibitor added to sulfonylurea monotherapy. In this study, short-term efficacy observed at 24 weeks was maintained over the longer term. Overall, treatment with dapagliflozin improved glycemic control, body weight, and blood pressure over a total of 48 weeks in patients whose T2DM was inadequately controlled on glimepiride. Although hypoglycemic events and events suggestive of genital infections were reported more often in patients receiving dapagliflozin, therapy was well-tolerated in general over 48 weeks. Given the common use of sulfonylureas in the management of T2DM in a number of countries, sustained efficacy and long-term tolerability with add-on therapies of complementary mechanisms of action such as dapagliflozin are important to establish.

\section{ACKNOWLEDGMENTS}

This study and article processing charges were funded by AstraZeneca and Bristol-Myers Squibb. We thank Simon Lancaster of inScience Communications, Springer Healthcare, for medical writing and editorial assistance. Support for this assistance was funded by AstraZeneca and Bristol-Myers Squibb. This study was previously presented at the International Diabetes Federation (IDF) 21st World Diabetes Congress, December 4-8, 2011, Dubai, United Arab Emirates. All named authors meet the ICMJE criteria for authorship for this manuscript, take responsibility for the integrity of the work as a whole, and have given final approval to the version to be published. All authors had full access to all of the data in this study and take complete responsibility for the integrity of the data and accuracy of the data analysis.

Conflict of interest. Dr. Strojek has received honoraria for speaking engagements, participation in advisory boards and clinical trials from Abbott, AstraZeneca, Bayer, Boehringer Ingelheim, Bristol-Myers Squibb, Eli Lilly, Merck Serono, Novartis, Novo Nordisk, Sanofi-Aventis, and Servier. Dr. Yoon has served on an advisory boards and participated in clinical trials programs for AstraZeneca, Eli Lilly, Merck, and Pfizer, and has received research support from Merck, AstraZeneca, and Bayer. Dr. Hruba was an employee of AstraZeneca at the time of the study and manuscript development. Ms. Sugg is an employee of AstraZeneca. Dr. Langkilde is an employee of AstraZeneca. Dr. Parikh is an employee of AstraZeneca.

Compliance with ethics guidelines. All procedures followed were in accordance with the ethical standards of the responsible committee on human experimentation (institutional and national) and with the Helsinki Declaration of 1975, as revised in 2000 and 2008. Informed consent was obtained from all patients for being included in the study.

Open Access. This article is distributed under the terms of the Creative Commons Attribution Noncommercial License which permits any noncommercial use, distribution, and reproduction in any medium, provided the original author(s) and the source are credited.

\section{REFERENCES}

1. Kahn SE, Haffner SM, Heise MA, et al. Glycemic durability of rosiglitazone, metformin, or glyburide monotherapy. N Engl J Med. 2006;355:2427-43. 
2. Nathan DM, Buse JB, Davidson MB, et al. Medical management of hyperglycemia in type 2 diabetes: a consensus algorithm for the initiation and adjustment of therapy: a consensus statement of the American Diabetes Association and the European Association for the Study of Diabetes. Diabetes Care. 2009;32:193-203.

3. Prospective UK. Diabetes Study Group. U.K. Prospective Diabetes Study 16. Overview of 6 years' therapy of type II diabetes: a progressive disease. U.K. Prospective Diabetes Study Group. Diabetes. 1995;44:1249-58.

4. Meng W, Ellsworth BA, Nirschl AA, et al. Discovery of dapagliflozin: a potent, selective renal sodiumdependent glucose cotransporter 2 (SGLT2) inhibitor for the treatment of type 2 diabetes. J Med Chem. 2008;51:1145-9.

5. List JF, Woo V, Morales E, Tang W, Fiedorek FT. Sodium-glucose cotransport inhibition with dapagliflozin in type 2 diabetes. Diabetes Care. 2009;32:650-7.

6. Komoroski B, Vachharajani N, Boulton D, et al. Dapagliflozin, a novel SGLT2 inhibitor, induces dose-dependent glucosuria in healthy subjects. Clin Pharmacol Ther. 2009;85:520-6.

7. Komoroski B, Vachharajani N, Feng Y, Li L, Kornhauser D, Pfister M. Dapagliflozin, a novel, selective SGLT2 inhibitor, improved glycemic control over 2 weeks in patients with type 2 diabetes mellitus. Clin Pharmacol Ther. 2009;85:513-9.

8. Strojek K, Yoon KH, Hruba V, Elze M, Langkilde AM, Parikh S. Effect of dapagliflozin in patients with type 2 diabetes who have inadequate glycaemic control with glimepiride: a randomized, 24-week, double-blind, placebo-controlled trial. Diabetes Obes Metab. 2011;13:928-38.

9. Inzucchi SE, Bergenstal RM, Buse JB, et al. Management of hyperglycemia in type 2 diabetes: a patient-centered approach: position statement of the American Diabetes Association (ADA) and the European Association for the Study of Diabetes (EASD). Diabetes Care. 2012;35:1364-79.

10. Zhang $\mathrm{M}$, Tsiatis AA, Davidian M. Improving efficiency of inferences in randomized clinical trials using auxiliary covariates. Biometrics. 2008;64:707-15.

11. Bolinder J, Ljunggren O, Kullberg J, et al. Effects of dapagliflozin on body weight, total fat mass, and regional adipose tissue distribution in patients with type 2 diabetes mellitus with inadequate glycemic control on metformin. J Clin Endocrinol Metab. 2012;97:1020-31.
12. Nauck MA, Del Prato S, Meier JJ, et al. Dapagliflozin versus glipizide as add-on therapy in patients with type 2 diabetes who have inadequate glycemic control with metformin: a randomized, 52-week, double-blind, active-controlled noninferiority trial. Diabetes Care. 2011;34:2015-22.

13. Wilding JP, Woo V, Soler NG, et al. Long-term efficacy of dapagliflozin in patients with type 2 diabetes mellitus receiving high doses of insulin: a randomized trial. Ann Intern Med. 2012;156:405-15.

14. Mitri J, Hamdy O. Diabetes medications and body weight. Expert Opin Drug Saf. 2009;8:573-84.

15. Lewington S, Clarke R, Qizilbash N, Peto R, Collins R. Age-specific relevance of usual blood pressure to vascular mortality: a meta-analysis of individual data for one million adults in 61 prospective studies. Lancet. 2002;360:1903-13.

16. Liu L, Zhang Y, Liu G, Li W, Zhang X, Zanchetti A. The Felodipine Event Reduction (FEVER) Study: a randomized long-term placebo-controlled trial in Chinese hypertensive patients. J Hypertens. $2005 ; 23: 2157-72$.

17. UK Prospective Diabetes Study Group. Tight blood pressure control and risk of macrovascular and microvascular complications in type 2 diabetes: UKPDS 38. BMJ. 1998;317:703-13.

18. Patel A, MacMahon S, Chalmers J, et al. Effects of a fixed combination of perindopril and indapamide on macrovascular and microvascular outcomes in patients with type 2 diabetes mellitus (the ADVANCE trial): a randomised controlled trial. Lancet. 2007;370:829-40.

19. Sowers JR, Epstein M, Frohlich ED. Diabetes, hypertension, and cardiovascular disease: an update. Hypertension. 2001;37:1053-9.

20. Bertoia ML, Waring ME, Gupta PS, Roberts MB, Eaton CB. Implications of new hypertension guidelines in the United States. Hypertension. 2012;60:639-44.

21. AstraZeneca. Multicenter Trial to Evaluate the Effect of Dapagliflozin on the Incidence of Cardiovascular Events (DECLARE-TIMI58). http://clinicaltrials.gov/ show/NCT01730534 (Accessed 26 March 2014).

22. Marre M, Shaw J, Brandle $M$, et al. Liraglutide, a once-daily human GLP-1 analogue, added to a sulphonylurea over 26 weeks produces greater improvements in glycaemic and weight control compared with adding rosiglitazone or placebo in subjects with Type 2 diabetes (LEAD-1 SU). Diabet Med. 2009;26:268-78. 
23. Muller LM, Gorter KJ, Hak E, et al. Increased risk of common infections in patients with type 1 and type 2 diabetes mellitus. Clin Infect Dis. 2005;41:281-8.

24. Shah BR, Hux JE. Quantifying the risk of infectious diseases for people with diabetes. Diabetes Care. 2003;26:510-3.

25. Bohannon NJ. Treatment of vulvovaginal candidiasis in patients with diabetes. Diabetes Care. 1998;21:451-6.

26. Drivsholm $\mathrm{T}$, deFineOlivarius $\mathrm{N}$, Nielsen $\mathrm{AB}$, Siersma V. Symptoms, signs and complications in newly diagnosed type 2 diabetic patients, and their relationship to glycaemia, blood pressure and weight. Diabetologia. 2005;48:210-4.

27. Joshi N, Caputo GM, Weitekamp MR, Karchmer AW. Infections in patients with diabetes mellitus. N Engl J Med. 1999;341:1906-12.

28. Bailey CJ, Gross JL, Pieters A, Bastien A, List JF. Effect of dapagliflozin in patients with type 2 diabetes who have inadequate glycaemic control with metformin: a randomised, double-blind, placebo-controlled trial. Lancet. 2010;375:2223-33.

29. Ferrannini E, Jimenez Ramos S, Salsali A, Tang W, List JF. Dapagliflozin monotherapy in type 2 diabetic patients with inadequate glycemic control by diet and exercise: a randomized, double-blind, placebo-controlled, phase III trial. Diabetes Care. 2010;33:2217-24.

30. Rosenstock J, Aggarwal N, Polidori D, et al. Doseranging effects of canagliflozin, a sodium-glucose cotransporter 2 inhibitor, as add-on to metformin in subjects with type 2 diabetes. Diabetes Care. 2012;35:1232-8.

31. Bhogale A, Patel N, Sarpotdar P, et al. Systematic investigation on the interaction of bovine serum albumin with $\mathrm{ZnO}$ nanoparticles using fluorescence spectroscopy. Colloids Surf B Biointerfaces. 2013;102:257-64.

32. Patel N, Taveira TH, Choudhary G, Whitlatch H, Wu WC. Fasting serum C-peptide levels predict cardiovascular and overall death in nondiabetic adults. J Am Heart Assoc. 2012;1:e003152.

33. Johnsson KM, Ptaszynska A, Schmitz B, Sugg J, Parikh SJ, List JF. Urinary tract infections in patients with diabetes treated with dapagliflozin. J Diabetes Complicat. 2013;27:473-8.

34. Johnsson KM, Ptaszynska A, Schmitz B, Sugg J, Parikh SJ, List JF. Vulvovaginitis and balanitis in patients with diabetes treated with dapagliflozin. J Diabetes Complicat. 2013;27:479-84.

35. Abdul-Ghani MA, Merovci A, Solis-Herrera C, et al. Plasma glucose reduction with the SGLT2 inhibitor, dapagliflozin, improves insulin sensitivity and insulin secretion in T2DM. Diabetes. 2013;62(Supplement 1):A1-98 (242-OR).

36. Ptaszynska A, Johnsson K, Apanovitch AM, Sugg J, Parikh S, List J. Safety of dapagliflozin in clinical trials for T2DM. Diabetes. 2012; 61(Supplement):A258 (1011-P). 